Editorial

\title{
New modalities for interactive entertainment
}

Interaction technologies have recently become more and more robust, ubiquitous, and widespread. Their advances encompass both the enabling technologies (e.g., faster computer architectures and network communications, more powerful real-time video and audio processing, and so on) and the concept itself guiding research and development. Keywords such as user centric media, user-centered design, and human-centered computing [1-3] are emerging as novel directions for research. The focus on the user and on her capabilities reverts the original machine-centred paradigm and opens new perspectives for interactive entertainment.

Several other application domains can benefit from such an evolution, e.g., interactive experience of cultural heritage, therapy and rehabilitation, education, technologies for social inclusion, and so on. Entertainment, however, is one of the fields that can best take advantage of novel interaction technologies and of such a shift of paradigm. Consider, for example, the availability of low-cost input devices (e.g., Kinect, Wii), of techniques for synchronised analysis of multiple input modalities (speech, face, full-body gesture, biometric signals) derived from models of human perception, of sophisticated interaction metaphors and strategies taking into account human perception, cognition, and emotion, of advanced rendering technologies (3D audio, video, and graphics).

This special issue collects contributions that witness such a trend toward a novel human-centered interactive entertainment. The special issue gathers selected, extended contributions from the Intetain 2011 Conference, with a special focus on research concerning intelligent systems, techniques, and applications for interactive entertainment. Since 2005, the Intetain conferences provide a unique international forum for researchers in the field of interaction technologies, with a special focus on entertainment. The conference aims at enhancing the understanding of recent and anticipated advances in interactive technologies, and their applications to entertainment, education, culture, and the arts. In this tradition, Intetain 2011 - held in Genova, Italy, May 2011 - included the presentation of research works on virtual/mixed/augmented reality, hardware technologies and devices for entertainment, animation and virtual characters, non-verbal full body interaction, storytelling, affective user interfaces, and techniques for social interaction.

A first paper in this special issue shows how novel low-cost input devices, such as Kinect, have a significant impact on advanced user-centered entertainment applications. The paper presents a framework for controlling the navigation of a quadrotor in GPSdenied environments, exploiting a new Kinect-based natural user interface to manually control the navigation of the quadrotor.

Two papers focus on the output of entertainment systems in form of virtual characters. The first one introduces AsapRealizer, a novel platform for controlling virtual humans. It combines and extends previous Behaviour Realizers, and can be adapted to suit the needs of a particular application in broader scenarios. The second one proposes a novel approach to automatic generation of character animations drawing inspiration from techniques for construction of virtual agents. Given the specification of a set of high-level goals, animation is automatically produced through the generation of a sequence of actions, the translation of actions into animation commands, and the display of an animated scene through a 3D graphic engine.

Finally, three papers provide concrete examples of application of entertainment technologies in specific domains. One of them concerns Brain-Computer Interfaces (BCI), an emerging and promising research field. It focuses on the user experience of multiplayer $\mathrm{BCI}$ games by comparing the $\mathrm{BCI}$ approach with more traditional interaction technologies.

The second paper addresses active listening of sound and music content, a new paradigm for entertainment with music: real-time analysis of full-body movement and of social interaction enables users to actively mould music as they listen to it, creating and sharing their experience in a novel way.

The third one presents an Interactive Multimodal Environment (IME) to be used at school for educational purposes. In such an environment, the user augments her real experience with auditory and/or visual content, in an approach combining entertainment with education. The environment was used to practice listening to English as a Second Language and to enable children with severe disabilities to perform an interactive listening.

Whereas covering only partially a huge research and application area, these works are exemplary case studies that well demonstrate the potential impact of novel interaction technologies and paradigms for entertainment computing.

\section{References}

[1] I. Laso-Ballesteros, P. Daras (Eds.), User Centric Future Media, EU Commission, Sep. 2008.

[2] ISO 9241-210:2010, Ergonomics of Human-System Interaction - Part 210: Human-Centred Design for Interactive Systems, International Organization for Standardization (ISO), 2010.

[3] A. Jaimes, N. Sebe, D. Gatica-Perez, Human-centered computing: a multimedia perspective, Proceedings of the 14th Annual ACM International Conference on Multimedia, ACM Press, Santa Barbara, CA, USA, 2006, pp. 855-864.

Gualtiero Volpe Casa Paganini-InfoMus, University of Genova, Italy

Dennis Reidsma HMI, University of Twente, The Netherlands

Antonio Camurri Casa Paganini-InfoMus, University of Genova, Italy

Anton Nijholt HMI, University of Twente, The Netherlands 